\title{
Plurale Einführung in die VWL? Zu den Gestaltungsmöglichkeiten pluraler Einführungsveranstaltungen
}

\section{Sebastian Thieme}

\section{Zusammenfassung}

Mit dem Verweis auf einen umfangreichen Kanon wirtschaftswissenschaftlichen Wissens wird seitens etablierter Fachvertreterinnen und -vertreter der (Standard-)Ökonomik häufig argumentiert, dass dieser keinen Raum lasse, um Perspektiven der Pluralen Ökonomik in entsprechende Lehrveranstaltungen zu integrieren. Dagegen lässt sich anführen, dass z. B. Einführungslehrbücher der VWL mehrere 100 Seiten umfassen und der dort präsentierte Inhalt daher bereits aus praktischen Gründen für die Lehre reduziert werden muss. Vor diesem Hintergrund und basierend auf Erfahrungen mit einer Einführungsveranstaltung in die VWL werden im vorliegenden Beitrag Gestaltungsmöglichkeiten diskutiert, die auch in bestehenden Curricula genutzt werden können, um den Lehrveranstaltungen zur Ökonomik eine kritische Perspektive im Sinne der Pluralen Ökonomik zu ermöglichen.

\section{Schlüsselwörter}

Kritik $\cdot$ Kontroversität $\cdot$ Normativität $\cdot$ Plurale Ökonomik $\cdot$ Sozialwissenschaftliche Ökonomik 


\section{Entstehungskontext: Der umfangreiche VWL- Kanon als Argument gegen eine Pluralisierung?}

Wer die jüngere Debatte um eine Pluralisierung der Ökonomik ab dem Jahr 2000 verfolgt, wird eine Zäsur dahin gehend feststellen, dass der Zustand der Ökonomik Gegenstand wissenschaftlicher Untersuchungen geworden ist, deren Ergebnisse seit einigen Jahren vorliegen. ${ }^{1}$ Während also noch vor über zehn Jahren (2009) die Vertreterinnen und Vertreter der Standardökonomik die Kritik am Zustand der Ökonomik schlichtweg ignorieren konnten, verneinten oder mit Anekdoten relativierten, lassen sich die eben erwähnten empirischen Befunde der Pluralismus-Forschung heute nicht vom Tisch wischen. Das schlägt sich auch in der Argumentation nieder, wenn eine Pluralisierung nicht mehr kategorisch als studentische Befindlichkeit abgelehnt wird, sondern Standard-Ökonominnen und -Ökonomen zwar mit dem Anliegen der Studierenden sympathisieren, aber dann zu Protokoll geben, dass der Kanon an volkswirtschaftlichen Grundlagen so groß ist, dass eben kein Platz mehr für andere Dinge bliebe. ${ }^{2}$ Interessanterweise kann dieser Umstand aber eine irenische Brücke zwischen Pluraler Ökonomik und offenen Vertreterinnen und Vertretern der Standardökonomik bilden. Denn wenn der ohnehin umfangreiche Stoff der Standardökonomik für die Lehre reduziert werden muss, warum dann nicht generell auch den Kanon kritisch durchleuchten und auf ein entsprechendes Maß verringern? Gerade wenn Hochschullehre im Sinne des Humboldtschen Ideals der Einheit von Forschung und Lehre gesehen wird, sollten doch auch Vertreterinnen und Vertreter der Standardökonomik mit Interesse darauf schauen, auf was sich von heute (noch) vorhandenen Lehrinhalten verzichten lässt, um somit Raum dafür zu schaffen, sich mit den eigenen Forschungsinteressen und -ergebnissen verstärkt in die Lehre einzubringen.

Thematisch liefert der vorliegende Beitrag in diesem Kontext einen Erfahrungsbericht darüber, ob und inwiefern standardökonomische VWL-Einführungsveranstaltungen plural ausgestaltet werden können. Meine Überlegungen basieren auf den Erfahrungen, die ich an einer deutschen Hochschule für angewandte Wissenschaften (HAW) in einem verwaltungswissenschaftlichen Studiengang (Bachelor) sammeln konnte. Dabei handelt es sich um

\footnotetext{
${ }^{1}$ Siehe unter anderem die (erste) sozialökonomische Analyse zur Entwicklung der Heterodoxie in Deutschland von Heise et al. (2017), aber auch die zahlreichen Lehrbuch-Analysen - z. B. von Rebhan (2017), Peukert (2018a), Peukert (2018b) und van Treeck und Urban (2016).

${ }^{2}$ Darauf läuft z. B. der Beitrag von Becker (2017) hinaus.
} 
die mikroökonomisch geprägte Veranstaltung „Grundlagen der Volkswirtschaftslehre“. Mit diesem Erfahrungsbericht ziele ich auf den alltäglichen Zustand ab, mit dem sich die Lehrenden an vielen deutschen Hochschulen konfrontiert sehen mögen. Das Thema dieses Beitrags dreht sich also dezidiert um das Ausloten von Möglichkeiten im Rahmen bestehender Curricula.

\section{Lehrinnovation am Praxisbeispiel „Einführung in die Volkswirtschaftslehre"}

Zur Beantwortung der Frage, wie sich standardökonomische Einführungsveranstaltungen mit alternativen Lehrinhalten ergänzen lassen, ist es zweckmäßig, zunächst darüber nachzudenken, welchem Ziel die VWL-Einführungsveranstaltungen folgen sollen. Wer entsprechende Lehrbücher konsultiert, wird sich des Eindrucks nicht erwehren können, dass deren Umfang und Struktur dazu anregen, sich in aller Regel mit einem Modell (z. B. Angebot-NachfrageModell) an bestimmten Themengebieten (Geld, Arbeitsmarkt, Entscheidungen usw.) abzuarbeiten. Dem mag die Intention zugrunde liegen, mit diesem Vorgehen z. B. das Systemverständnis zu stärken und ebenso die Fähigkeit zu trainieren, ökonomische Zusammenhänge durch die systematische (analytische) Betrachtung von Kernprozessen zu durchdringen. Insofern ist es auch nicht verwunderlich, wenn diese Lehrbücher eine Lehrpraxis nahelegen, die im Abarbeiten der dort dargebotenen Inhalte besteht. Das mag dann vor allem angesichts unterfinanzierter Hochschulen als gangbarer Weg empfunden werden. Schwierig ist aber, dass mit diesen Lehrbüchern bereits ein vorgefasstes spezifisches Verständnis von Wirtschaft und Wirtschaftswissenschaft zugrunde gelegt wird und dann auch kaum (mehr) der Raum existiert, um dieses Verständnis und die Methodik kritisch - im Vergleich mit Alternativen - zur Disposition zu stellen. Insofern ist auch der Vorwurf der Einseitigkeit zu verstehen, der seitens der Pluralen Ökonomik erhoben wird.

Aus Sicht der Pluralen Ökonomik sollte das Ziel der Lehre sein, die Bandbreite an wissenschaftlichen Zugängen, Fragen usw. zu vermitteln. Dies gewinnt an Bedeutung, wenn Standards guter Lehre hinzugezogen werden, wie z. B. das Kontroversitätsgebot im Rahmen des Beutelsbacher Konsens. ${ }^{3}$ Doch geht es

\footnotetext{
${ }^{3}$ Im Beutelsbacher Konsens sind in den 1970er Jahren drei Leitprinzipien der politischen Bildung formuliert worden, die bis heute als Referenzpunkt für die Schulbildung (und darüber hinaus) gelten: 1) Das Überwältigungsverbot besagt, dass die Lernenden nicht
} 
dabei nicht nur um die inhaltliche Vielfalt, sondern auch um die Frage, welches Bild der Ökonomik in den Einführungsveranstaltungen vermittelt wird. Das ist deshalb eine wichtige Frage, weil solche Einführungsveranstaltungen oft auch von Studierenden anderer Studienfächer besucht werden, um einen Überblick zur Ökonomik zu erhalten. Einführungsveranstaltungen sollten daher an dem Ziel ausgerichtet sein, nicht den Eindruck einer quasi einheitlichen und widerspruchsfreien ,neoklassischen“ Modellökonomik zu vermitteln, sondern das Interesse für die Vielfalt an Fragen, Methoden, Modellen, Kontroversen usw. zu wecken. Nicht zuletzt dadurch werden auch Grundlagen für Interdisziplinarität gelegt. Allerdings stellt sich hierbei das gleiche Problem, mit dem Standard-Ökonominnen und -Ökonomen angesichts eines großen Kanons an volkswirtschaftlichen Grundlagen konfrontiert sind: Der Bereich der Pluralen Ökonomik ist so breit, dass auch dort eine Eingrenzung erfolgen muss, weil in einer Einführung nicht alles und vieles nicht im Detail behandelt werden kann.Als Mittelweg kann die Methode des weiter unten vorgestellten Drei-Punkt-Schemas angewendet werden, das auf eine kritische Einführungsveranstaltung hinausläuft, die i) standardökonomische Inhalte lehrt, ii) diese kritisiert und iii) Alternativen aufzeigt. Das wird nicht ohne Eingrenzung der Lehrinhalte und Konzentration auf ausgewählte Inhalte gehen. Doch wie lassen sich solche Abstriche rechtfertigen? Dazu lässt sich zunächst der genannte Punkt anführen, dass der - seitens der Pluralen Ökonomik als „einseitig“ kritisierte - Standard-VWL-Kanon ohnehin einen Umfang besitzt, der kaum in einem Semester zu bewältigen und daher einzugrenzen ist. Zweitens lässt sich generell infrage stellen, ob es überhaupt Ziel einer Einführungsveranstaltung sein muss, einen Kanon zu lehren. Sollte die Kenntnis des Kanons nicht Ergebnis der gesamten Ausbildung sein, die sich über verschiedene Lehrveranstaltungen erstreckt? Gleiches lässt sich für die Perspektive der Pluralen Ökonomik geltend machen: Auch hier muss eine Einführungsveranstaltung nicht die ganze Bandbreite an Ansätzen, Methoden usw. darstellen. Diese könnten, sofern es das Lehrangebot zulässt, später in anderen

an der selbstständigen Bildung eines eigenen Urteils gehindert werden sollen, d. h. sie sollen nicht indoktriniert werden. 2) Das Kontroversitätsgebot hält dazu an, unterschiedliche Perspektiven einzubringen, keine Option zu unterschlagen. 3) Die Lernenden sollen zu politischem Handeln befähigt werden, d. h. eine politische Situation und die eigene Interessenlage analysieren können sowie in der Lage sein, eigenständig nach Möglichkeiten zu suchen, mit denen sich die politische Situation im Sinne der eigenen Interessen beeinflussen lässt. Siehe dazu Bundeszentrale für politische Bildung (2011). 
Veranstaltungen ergänzt und vertieft werden. Insofern lässt sich bezogen auf den Inhalt von Einführungsveranstaltungen argumentieren, dass so, wie die Standardökonomik später vertieft werden kann, die Einführungsveranstaltungen dazu einladen sollen, später auch alternative Zugänge zu ökonomischen Fragen zu vertiefen. Vor diesem Hintergrund kann , der Mut zur Lücke‘ praktiziert werden, der vom Bemühen um die Präsentation eines möglichst vollständigen Kanons an VWL-Wissen befreit und stattdessen motivational eine Einführungsveranstaltung als das begreift, was sie ist: Eine Vermittlung basaler Grundbegriffe und Techniken sowie ein darüber hinaus reichender Überblick über unterschiedliche Themen, Perspektiven, Diskurse und wissenschaftliche Verfahren. Die im Beutelsbacher Konsens geforderte Kontroversität kann sich damit auf unterschiedliche Positionen und Darstellungen innerhalb der Standardökonomik, der Kritik daran und ausgewählte alternative Ansätze konzentrieren. Damit wäre dem Beutelsbacher Konsens (Bundeszentrale für politische Bildung 2011) auch im Sinne des Überwältigungsverbots gedient sowie im Sinne des Anspruchs, die Studierenden zur eigenständigen Analyse zu befähigen.

Nun mag eingewendet werden, dass, wenn Einführungsveranstaltungen sich an disziplinär heterogene Studierende richten und Inhalte einzugrenzen sind, dann auch das Niveau der Lehrveranstaltungen in entsprechend angemessener Weise angepasst werden müsse: Gemeint wäre damit vor allem der Grad der formal-mathematischen Darstellungen. ${ }^{4}$ Dagegen lassen sich zwei Argumente ins Feld führen. Erstens spricht viel für den Standpunkt, dass die Einführungsveranstaltungen tatsächlich nur einen ersten Einblick liefern und grundsätzliche Lehrmeinungen bzw. Zusammenhänge vermitteln sollen. Alles das, was dann über einen gewissen Grad an formaler Modellökonomik hinaus nötig wird, wäre in vertiefenden Veranstaltungen und Seminaren besser aufgehoben. Das heißt nicht, dass Mensch auf Mathematik verzichten muss. So führt auch in Einführungsveranstaltungen kein Weg an Grenzkosten und Elastizitäten vorbei. Doch wie hoch soll der Grad der Formalisierung darüber hinaus sein? Diese Frage führt - zweitens - zu (standardökonomischen) Lehrbüchern wie Krugman und Wells (2017), die zeigen, dass mit verhältnismäßig wenig formal-

\footnotetext{
${ }^{4}$ Dahinter mag sich seitens der Standardökonomik die Befürchtung verbergen, das Niveau absenken zu müssen. Allerdings kann das auch ein gepflegter Vorbehalt sein, denn möglicherweise sind „Fachfremde“ auch oder gar besser in der Lage, die formalmathematischen Darstellungen zu verstehen. Mensch denke an Studierende der Ingenieurwissenschaften, Mathematik oder Lehramtsstudierende mit Zweitfach Mathematik.
} 
mathematischem Aufwand auch standardökonomische Inhalte vermittelt werden können, ohne dass jemand auf die Idee kommen müsste, dort würde Ökonomik ohne Mathematik betrieben und lediglich imitiert werden. Abschließend ist es mir wichtig, darauf hinzuweisen, dass die Vermittlung eines Überblicks zur wirtschaftswissenschaftlichen Landschaft selbstverständlich und notwendigerweise auch die Standardökonomik umfassen muss. Notwendigerweise deshalb, weil es das dominante Wissen ist, mit denen die Studierenden üblicherweise konfrontiert sind, wenn sie auf Ökonominnen und Ökonomen stoßen. Es wäre aus rein praktischen Gründen verfehlt, den Studierenden eine real-world-Einführung in die VWL anzubieten, in der die kritisierte Standardökonomik völlig außen vor bliebe. Denn dann wüssten die Studierenden im Alltag möglicherweise nicht, wie sie mit dem standardökonomischen Wissen umzugehen haben. Selbst an der Pluralen Ökonomik orientierte Studierende der Wirtschaftswissenschaften, aber auch jene Studierende aus benachbarten oder anderen Studiengängen wie z. B. Verwaltungsökonomie, Medienökonomik oder Soziologie, sollten in einer VWL-Einführung vermittelt bekommen, wie ,normal' (standardökonomisch) ausgebildete Ökonominnen und Ökonomen denken.

Wenn also die Standardökonomik zum Lehrinhalt gehören soll, in welchem Umfang und wie lässt sich dann eine alternative - gar kritische - Perspektive dazu in die Lehrveranstaltungen integrieren? Darauf wird nachfolgend einzugehen sein.

\section{$3 \quad$ Konzept und Vorgehen}

In den Wintersemestern 2018/2019 und 2019/2020 habe ich die „Einführung in die Volkswirtschaftslehre" (VWL) für Studierende von verwaltungswissenschaftlichen Studiengängen (Verwaltungsökonomik etc.) an einer Hochschule für Angewandte Wissenschaften (HAW) abgehalten. Diese wurde jeweils drei Mal wöchentlich in großen Hörsälen im Umfang von je 2 Semesterwochenstunden angeboten und insgesamt je Wintersemester von etwa 176 Studierenden besucht. Als Leistungsnachweis war eine Klausur zu schreiben (Umfang: 1 Stunde), die ich als Aufgaben-Pool stellte: Die Studierenden konnten völlig frei die Aufgaben wählen und auch so viele Aufgaben lösen, wie sie wollten. ${ }^{5}$ Insgesamt

\footnotetext{
${ }^{5}$ Es gab eine maximale Zahl an zu erreichenden Punkten. Wer diese überschritt, bekam trotzdem nur die maximale Zahl an Punkten angerechnet.
} 
standen zwölf bzw. elf Lehreinheiten zur Verfügung, für die ich mich an den Inhalten meines Vorgängers orientierte. Dieser verwendete das aktuelle Lehrbuch von Krugman und Wells (2017) und behandelte dort jeweils einzelne Kapitel (z. B. ökonomische Grundprinzipien, ökonomische Modelle, Externalitäten und Steuern). Insofern existierten bereits ein zeitlich-organisatorischer Rahmen und inhaltliche Ankerpunkte. Doch schon die ersten Kapitel von Krugman und Wells (2017) zeigten mir, dass die Inhalte nicht unkommentiert vermittelt werden sollten. ${ }^{6}$ Davon ausgehend entschied ich mich dem Kontroversitätsgebot folgend dazu, die Lehrveranstaltungen soweit wie möglich gemäß der folgenden DreiPunkte-Methodik (SKA) aufzubauen: i) standardökonomische Perspektive, ii) Kritik und iii) Alternativen. ${ }^{7}$ Bei der Darstellung der standardökonomischen Inhalte (i) habe ich mich zwar mit Schwerpunkt an Krugman und Wells (2017) orientiert, aber prinzipiell noch andere Lehrbücher konsultiert, um je nach Themengebiet nach alternativen Darstellungen $\mathrm{zu}$ schauen. Das war wichtig, um den Studierenden das Selbststudium zu erleichtern und ihnen verschiedene Zugänge zum Verständnis zu bieten. Dabei habe ich jenen Lehrbüchern den Vorzug gegeben, die über die Hochschul-Bibliothek auch leichter verfügbar waren. Und in der Tat fanden sich z. B. in Engelkamp und Sell (2011), Deimer et al. (2017) und Neubäumer et al. (2017) interessante Ergänzungen, die nicht alle notwendigerweise dem Bereich einer dezidiert heterodoxen Ökonomik zuzurechnen sind.

Für die kritische Perspektive und die Alternativen ( $i i+i i i)$ habe ich das Lehrbuch von Biesecker und Kesting (2003) als sehr hilfreich empfunden, dazu aber

${ }^{6}$ Z. B. wird von Krugman und Wells (2017, S. 6 ff.) die Meinung vertreten, dass Ökonominnen und Ökonomen über Anreize das Verhalten ändern sollen. Dazu wird ein Beispiel angeführt, in dem Kindern für schulische Leistungen Geld geboten wurde und diese dann bessere Leistungen nach Hause brachten. Mag die dazu angeführte Studie selbst über jeden Zweifel erhaben sein, so hätte es doch zumindest die wissenschaftliche Redlichkeit erfordert, auf den nicht unbedenklichen Verdrängungseffekt hinzuweisen, gemäß dem intrinsische Motivation (Freude, Neugierde usw.) durch externe Regulierung verdrängt wird. Dieser Effekt ist praktisch ein Allgemeinplatz in der Pädagogik und Psychologie, siehe dazu z. B. Deci und Ryan (1993).

${ }^{7}$ „Soweit wie möglich“ bezieht sich darauf, dass nicht zu jedem Thema zwangsläufig auch ein Kritikpunkt und/oder eine Alternative zu finden ist. Das gilt z. B. für das Thema „Elastizitäten“. Beim Thema „Steuern“ lag der Fall so, dass ich aus anderen Lehrbüchern die für Deutschland relevanten Aspekte (z. B. Arten der Abgaben) nachholen musste und es dazu kaum Kritikpunkte zur Vertiefung gab. 
- je nach Thema - weitere Literatur verwendet. Ich möchte das erwähnte DreiPunkte-Schema kurz an einem Beispiel illustrieren.

Im Kap. 1 von Krugman und Wells (2017) werden zwölf Grundprinzipien der Ökonomik vorgestellt und behandelt, z. B. dass Ressourcen knapp sind, das Opportunitätskostenprinzip, das Prinzip der Nutzenmaximierung oder die Effizienz von Märkten. Kritisch ließ sich den Ausführungen im Lehrbuch unter anderem entgegenhalten, dass

- „Gleichgewicht“ und „Effizienz“ dort im Grunde synonym verwendet werden, sodass die Aussagen einen tautologischen Charakter besitzen ${ }^{8}$.

Entgegen der Herleitung eines , natürlichen“ Hangs zum Tausch zumindest die aus der BWL bekannte „Make or Buy“-Entscheidung zu beachten wäre, ob überhaupt ,gehandelt" werden soll

- die Ausführungen sich auf ein rein positives Bild ,der" Marktwirtschaft konzentrierten und alternative Wirtschaftsformen (solidarisches Wirtschaften, Genossenschaften, Eigenarbeit usw.) ignorierten

- der Staat bzw. das Gemeinwesen lediglich auf die Funktion als ,Reparaturbetrieb` für Marktversagen reduziert wurde.

Der letzte Punkt war insbesondere deshalb brisant, weil die Lehrveranstaltung im Rahmen verwaltungswissenschaftlicher Studiengänge stattfand. Es gestaltete sich als schwer, Studierenden, die später einmal in staatlichen oder kommunalen Institutionen tätig sein werden, eine Ökonomik zu vermitteln, die ihrer zukünftigen Tätigkeit grundsätzlich abwertend gegenübersteht. Hinzu kommt, dass der Hinweis auf , den“ effizienten Markt wenig bei der Frage hilft, wie z. B. kommunal geführte Unternehmen gegründet und bewirtschaftet werden sollen. Hierzu bedarf es anderer wissenschaftlicher Zugänge. Genau das wurde dann im dritten Schritt über die sozial-ökologische Perspektive von Biesecker und Kesting (2003) kurz vorgestellt, wo neben der Marktökonomie auch die Versorgungsökonomie und der Dritte Sektor mit Non-Profit-Organisationen berücksichtigt wurden. Hinzu kamen das Konzept Vorsorgenden Wirtschaftens (Biesecker 2009)

\footnotetext{
${ }^{8}$ So schreiben Krugman und Wells (2017, S. 12): „Eine wirtschaftliche Situation ist im Gleichgewicht, wenn kein Individuum sich durch eine Änderung seines Verhaltens verbessern kann.“ Effizienz ist bei Krugman und Wells (2017, S. 14) wie folgt definiert: „Eine Volkswirtschaft ist dann effizient, wenn sie alle Möglichkeiten nutzt, um Menschen besser zu stellen, ohne dass andere Menschen schlechter gestellt werden“ (im Original mit Hervorhebung).
} 
sowie das safety-first principle im Rahmen der moral economy von Scott (1976) als Beispiele für alternative Grundprinzipien des Wirtschaftens.

Um dem Ziel einer besonders kontrovers gestalteten Lehrveranstaltung gerecht zu werden, bedarf es meiner Meinung nach der richtigen Kontrastpunkte. Damit meine ich Ansätze, die sich der sozialwissenschaftlichen Ökonomik zurechnen lassen (Thieme 2019). ${ }^{9}$ Mit ihrer methodisch und inhaltlich großen Differenz zur Standardökonomik sind sie besonders geeignet, die Forderungen nach einer Pluralen Ökonomik im Sinne nicht-formaler und sozialwissenschaftlicher Zugänge zu illustrieren. Damit sei keineswegs der heterodoxe Charakter von z. B. postkeynesianischen oder komplexitätsökonomischen Konzepten in Abrede gestellt. Doch wenn die Kritik der Pluralen Ökonomik an der Standardökonomik sich vor allem gegen die formal-mathematische Modellkultur, die Abstraktheit und den mangelnden sozialwissenschaftlichen Charakter richtet, werden Erwartungen geweckt, die eine wenn auch deutlich andere Modellökonomik nur in sehr begrenztem Umfang zu erfüllen vermag. Zudem sind es vor allem die Ansätze der sozialwissenschaftlichen Ökonomik, in denen z. B. die normativen Kritikpunkte an der standardökonomischen Perspektive besonders deutlich herausgearbeitet wurden. Eine gute Hilfestellung für eine solche Pluralisierung bzw. Kontroverse lieferte das Lehrbuch von Biesecker und Kesting (2003). Das Besondere dieses Buches liegt darin, dass dort Menschenbilder und Rationalitäten je nach wissenschaftlichem Zugang vorgestellt werden. Es wird außerdem betont, welche Rationalitätstypen sich eben nicht in eine typisch formal-mathematische Modellökonomik - sei sie nun heterodox oder standardökonomisch - einfügen. Wer beispielsweise später einmal im Bereich Gesundheit arbeiten wird, ist damit nicht auf eine technische KostenEffizienz-Rationalität im Sinne der Standardökonomik angewiesen, sondern weiß, dass es jenseits davon auch andere rationale Perspektiven des Wirtschaftens gibt.

Eine besondere Herausforderung für die Lehre stellt die Normativität der Ökonomik dar. Und zwar nicht allein deshalb, weil sich die oft postulierte Unterscheidung zwischen ,positiver“" und ,normativer“ Ökonomik aus guten Gründen kritisieren lässt. ${ }^{10}$ Sondern auch, weil diese in den (standardöko-

\footnotetext{
${ }^{9}$ Damit meine ich unter anderem feministische Ökonomiken, Alt-Institutionalismus, Sozialökonomik, den Bielefelder Subsistenzansatz, das Konzept Vorsorgenden Wirtschaftens, die Wirtschaftsstile oder die Integrative Wirtschaftsethik.

${ }^{10}$ Der Schwerpunkt der Kritik liegt vor allem auf der als verfehlt angesehenen Idee einer rein positiven Ökonomik. Typische Kritikpunkte sind: die Kritik am damit verbundenen Postulat der Wertfreiheit bei Thielemann (2015) und Ulrich (2008); die Kritik am ebenfalls damit verbundenen Wahrheitsanspruch sowie die Ignoranz gegenüber der Performativität der Ökonomik bei Hirte (2017); das, was Biesecker und Kesting (2003) als ,preanalytic
} 
nomischen) Lehrbüchern selbst postulierte Unterscheidung bisweilen unterlaufen wird. So erklären zwar Krugman und Wells (2017), dass ,die' Ökonomik eine „positive“ Ökonomik sei, schreiben dann aber, dass Ökonominnen und Ökonomen Annahmen usw. für Modelle selbst festlegen (ebd., S. 45 f.). Dass es sich dabei auch um Entscheidungen handelt, die begründungsbedürftig sein können und in diesem Sinne dann auch einen normativen (wissenschaftsethischen) Charakter besitzen, wird ausgeblendet. Das betrifft Unstimmigkeiten wie z. B. „naturalistische“ und damit nicht weiter begründete Annahmen (Nutzenmaximierung, Handel führt zu Vorteilen usw.); oder auch die Behauptung, es gäbe keine allgemein akzeptierte Definition von Gerechtigkeit und Fairness, der deutlich das Bemühen anzumerken ist, die Frage nach einer normativen Ökonomik möglichst schnell zu den Akten zu legen.Nun lässt sich in einer Einführungsveranstaltung nicht die ganze Bandbreite an normativen bzw. wirtschaftsethischen Überlegungen und Konzepten präsentieren. Es sollte aber ebenfalls klar sein, dass die oft in Lehrbüchern verächtlich gemachte normative Analyse ganz klar ihren Platz in der Ökonomik hat. Denn viele Fragen, mit denen sich Ökonominnen und Ökonomen konfrontiert sehen, berühren die normative Ebene (Entlohnung, Wohnungsmiete, Armut usw.). Daran ändert auch keine noch so kunstvoll gewundene Transformation von normativen Fragen in ,positive“ Fragen etwas. ${ }^{11}$ Was sich mit Blick auf die Normativität in der Ökonomik in Einführungsveranstaltungen durchaus realisieren lässt, das ist ein normativ-kritischer Blick auf das, was standardökonomisch behauptet wird. Dies lässt sich sehr gut mit dem zweiten Punkt der oben vorgestellten Drei-Punkt-Methodik (SKA) - der Kritik - verbinden. Entsprechend wurden in den Lehrveranstaltungen dort, wo es im Referenz-Lehrbuch eine Rolle spielte, die erwähnten begründungsbedürftigen Annahmen (z. B. ökonomische Rationalität und Nutzenmaximierung) kritisiert. Zweifelsohne sind damit aber auch entsprechende Anforderungen an das Lehrpersonal gestellt (Kenntnisse in Wissenschafts- und Erkenntnistheorie, Wirtschaftsethik usw.).

vision" bezeichnen, also der Umstand, dass alles Theoretisieren durch die eigenen Erfahrungen, das Umfeld, die Kultur, den Zeitgeist usw. geprägt ist.

${ }^{11}$ Ein typisches Beispiel dafür ist der Umgang mit Umweltfragen, die zu „Wie-viel“-Fragen umgewandelt werden, bei denen dann nicht etwa die Vermeidung von z. B. gesundheitsschädlichen Substanzen im Vordergrund steht, sondern die Bestimmung eines ,optimalen “ Verschmutzungsgrads. Die Gefahr liegt dabei darin, in ähnlicher Weise viele der als absolut geltenden Menschen- und Grundrechte (Wohnraum, Menschenwürde usw.) zur Disposition zu stellen. 


\section{$4 \quad$ Einschätzung und Wirkungen}

Für die inhaltliche Kontroversität der Lehrveranstaltungen ist es erstens hilfreich, standardökonomische Lehrbücher auszuwählen, die deutliche Kritik hervorrufen. Das war im Falle von Krugman und Wells (2017) gegeben, z. B. hinsichtlich der ökonomischen Prinzipien am Anfang des Lehrbuchs, der Unterscheidung zwischen „positiver“ und „,normativer“ Ökonomik, der Erläuterungen zur Allmende oder der Erklärungen für die Anwendung des Marginalitätsprinzips. Zweitens ist es für die Kontrastierung im Sinne einer Pluralen Ökonomik hilfreich, auf Alternativen zurückzugreifen, die sich deutlich von der Standardökonomik unterscheiden. Damit ziele ich auf den bereits erwähnten Bereich der kultur-, geistes- und sozialwissenschaftlichen Ökonomik ab, der unter anderem feministische Ökonomiken, Sozialökonomik und die Integrative Wirtschaftsethik umfasst (Thieme 2019). Drittens sollte darüber hinaus berücksichtigt werden, dass sich standardökonomische Darstellungen bisweilen von Lehrbuch zu Lehrbuch unterscheiden können. Daher kann es ebenfalls zur Kontroversität beitragen, mehrere Lehrbücher zu konsultieren und entsprechend unterschiedliche Darstellungen in die Lehrveranstaltung zu integrieren.

Der wesentlichste Punkt an einer kontrovers gestalteten Lehrveranstaltung ist aber, dass eine kontroverse Darstellung von Lehrinhalten nicht die Klarheit und Eindeutigkeit erzeugt, wie es von standardökonomischen Lehrbüchern üblicherweise geboten wird. Das bedeutet, dass die Studierenden lernen müssen, mit Unsicherheit umzugehen. Das mag die Studierenden im ersten Moment verschrecken. Hier hilft es einerseits, die Studierenden in den Veranstaltungen immer wieder daran zu erinnern, dass diese Unsicherheit ,normal ' ist. Das lässt sich vor allem dort gut realisieren, wo die Standardökonomik zu kritischen Fragen führt, weil das Alltagserleben oder andere Gründe gegen die standardökonomischen Lösungen sprechen. Andererseits sollten die Lehrenden die Studierenden nicht mit der Kritik alleine lassen. Zentral ist es deshalb, Alternativen aufzuzeigen, z. B. andere Studien oder alternative (heterodoxe) Theorien. Das hat wiederum seine, natürlichen' Grenzen im zur Verfügung stehenden Zeitbudget. Hilfreich kann es daher sein, ergänzende Vertiefungsseminare anzubieten. Tatsächlich verhielt es sich in meinem Falle so, dass Studierende immer wieder nach (mehr) Alternativen fragten, mit denen über die Kritik an der Standardökonomik hinausgegangen werden konnte. Einzelne davon nahmen ein zusätzliches Lehrangebot „Einführung in die Plurale Ökonomik in Deutschland“ gerne an.

Wie wurde die Lehrveranstaltung von den Studierenden aufgegriffen? In der entsprechenden Evaluation (WS 2018/2019) bewerteten die Studierenden die 
Veranstaltung als „gut“. Insbesondere der kritische Blickwinkel, aber auch die Orientierung am Standard-Lehrbuch wurden immer wieder als positiv angesehen. Die Meinungen zum Umfang des Skripts gingen bisweilen auseinander: Während die Lehrinhalte der einen Gruppe von Studierenden als umfangreich erschienen, wertete eine andere Gruppe von Studierenden diese als angemessen. ${ }^{12}$ Die Kolleginnen und Kollegen am Fachbereich Verwaltungswissenschaften waren einer solchen Einführung gegenüber sehr aufgeschlossen, was unter anderem an der ohnehin interdisziplinären und offenen Haltung der Lehrenden dort lag und ebenso am Interesse, eine auf die speziellen Bedürfnisse der Verwaltungswissenschaften abgestimmte Form der Ökonomik zu vermitteln. Gleichwohl verhielt es sich so, dass ein Engpass in der Lehre bestand und die Verantwortlichen froh waren, als ich die Lehre im Rahmen von Vertretungsprofessuren übernahm. Als institutionell gefestigt können diese Veranstaltungen leider nicht betrachtet werden, da sie eng mit meiner Person verbunden waren und die Lehrveranstaltungen dort ab voraussichtlich Ende Wintersemester 2019/2020 nicht mehr von mir weitergeführt werden.

\section{$5 \quad$ Fazit}

Ich hoffe, gezeigt zu haben, dass eine Pluralisierung der Ökonomik auch im Rahmen bestehender Verhältnisse (Curricula) möglich ist. Das Argument, es müsse ein umfangreicher Kanon an volkswirtschaftlichen Grundlagen gelehrt werden und deshalb sei in Einführungsveranstaltungen kein Platz für Inhalte einer Pluralen Ökonomik, überzeugt angesichts solcher Lehrerfahrungen nicht. Vielmehr scheint es eine genehme Ausflucht zu sein, um sich des Aufwands zu entledigen, der notwendig wäre, wenn über den sinnvollen Inhalt solcher Lehrveranstaltungen nachgedacht werden soll. Dessen ungeachtet befremdet es, wenn Lehrende einen Kanon propagieren, der heute kaum noch zu bewältigen ist und sie ihm auch noch tatenlos beim Anwachsen zusehen. Es scheint fast so, dass

\footnotetext{
${ }^{12}$ Abschließend ist darauf hinzuweisen, dass die Erstellung des Lehrmaterials, die Recherche, das Vergleichen verschiedener Lehrbücher usw. zwar einen Aufwand darstellt, der aber auch von Lehrenden an Universitäten (mit weniger Lehrverpflichtung als an einer HAW) zu bewältigen sein sollte. Hinzu kommt, dass es nicht das eine „heterodoxe“ oder ,plurale“ Lehrbuch gibt, das sich zum Kontrast oder als Alternative verwenden lässt. Außerdem werden einzelne Themen (z. B. Allmende) durch weitere Papiere, Bücher etc. zu ergänzen sein.
} 
dann das Unausweichliche hinausgeschoben werden soll, um nur nicht selbst die unangenehme Arbeit erledigen zu müssen, die Lehrinhalte auf ein sinnvolles Maß zu reduzieren. Natürlich muss letztlich entschieden werden, was inhaltlich eine Berücksichtigung finden soll und was nicht. Damit setzen sich Lehrende immer auch der Kritik aus. Interessierte Hochschullehrende können dies aber als Ansatzpunkt nutzen, um Raum auch für alternative Inhalte zu schaffen. Dies ist durchaus möglich. Wo die auch didaktischen Schwierigkeiten liegen, darüber wird noch intensiver zu reden sein. Der vorliegende Beitrag sollte dazu Impulse liefern.

\section{Literatur}

Becker, J. (2017). Das „richtige“ Maß an Pluralität und das Problem des fehlenden Adressaten. Wirtschaftsdienst, 97, 835-853. https://blog.zeit.de/herdentrieb/ files/2017/12/wirtschaftsdienst_12-2017_Pluralismus-in-der-\%C3\%96konomik.pdf. Zugegriffen: 07. Sept. 2019.

Biesecker, A. (2009). Vorsorgendes Wirtschaften als Alternative. In Friedrich-EbertStiftung (Hrsg.), Antworten aus der feministischen Ökonomie auf die globale Wirtschafts- und Finanzkrise. Tagungsdokumentation der Friedrich-Ebert-Stiftung (S. 32-48). Bonn: Friedrich-Ebert-Stiftung. http://library.fes.de/pdf-files/wiso/0675320091109.pdf. Zugegriffen: 14.Okt. 2018.

Biesecker, A. \& Kesting, S. (2003). Mikroökonomik. Eine Einführung aus sozial-ökologischer Perspektive (Reprint 2014). Berlin: De Gruyter.

Bundeszentrale für politische Bildung. (2011). Beutelsbacher Konsens. https://www.bpb. de/die-bpb/51310/beutelsbacher-konsens. Zugegriffen: 08. Aug..2019.

Deci, E. L., \& Ryan, R. M. (1993). Die Selbstbestimmungstheorie der Motivation und ihre Bedeutung für die Pädagogik. Zeitschrift für Pädagogik 36, 223-238. https:// www.pedocs.de/volltexte/2017/11173/pdf/ZfPaed_1993_2_Deci_Ryan_Die_Selbstbestimmungstheorie_der_Motivation.pdf. Zugegriffen: 23. Aug. 2019.

Deimer, K., Pätzold, M., \& Tolkmitt, V. (2017). Ressourcenallokation, Wettbewerb und Umweltökonomie. Wirtschaftspolitik in Theorie und Praxis (1. Aufl.). Berlin: Springer.

Engelkamp, P., \& Sell, F. L. (2011). Einführung in die Volkswirtschaftslehre (5. Aufl.). Berlin: Springer.

Heise, A., Sander, H., \& Thieme, S. (2017). Das Ende der Heterodoxie? Die Entwicklung der Wirtschaftswissenschaften in Deutschland. Wiesbaden: Springer.

Hirte, K. (2017). Zur Performativität in den Wirtschaftswissenschaften: Kernaussagen, Anwendungspotenziale und Grenzen eines Konzepts. In R. Pfriem, U. Schneidewind, J. Barth, S. Graupe, \& T. Korbun (Hrsg.), Transformative Wirtschaftswissenschaft im Kontext nachhaltiger Entwicklung (S. 139-164). Marburg: Metropolis.

Krugman, P., \& Wells, R. (2017). Volkswirtschaftslehre (2. Aufl.). Stuttgart: Schäffer Poeschel.

Neubäumer, R., Hewel, B., Lenk, T., \& Egerer, E. (Hrsg.). (2017). Volkswirtschaftslehre. Grundlagen der Volkswirtschaftstheorie und Volkswirtschaftspolitik (6. Aufl.). Wiesbaden: Springer Fachmedien Wiesbaden. 
Peukert, H. (2018a). Makroökonomische Lehrbücher: Wissenschaft oder Ideologie? (1. Aufl.). Marburg: Metropolis.

Peukert, H. (2018b). Mikroökonomische Lehrbücher: Wissenschaft oder Ideologie? Marburg: Metropolis.

Rebhan, C. (2017). Einseitig oder plural? Eine quantitative Analyse der wirtschaftswissenschaftlichen Einführungslehrbücher an deutschen Hochschulen. Marburg: Metropolis.

Scott, J. C. (1976). The moral economy of the peasant. Rebellion and subsistence in Southeast Asia. New Haven: Yale University Press.

Thielemann, U. (2015). Integrative Wirtschaftsethik oder: Das Ganze des Wirtschaftens denken, und zwar kritisch. In T. Beschorner, P. Ulrich \& F. Wettstein (Hrsg.), St. Galler Wirtschaftsethik. Programmatik, Positionen, Perspektiven (S. 175-218). Marburg: Metropolis.

Thieme, S. (2019). Die Ökonomik als Sozialwissenschaft? Kritische Anmerkungen zur Pluralismus-Debatte. In S. Graupe, W. O. Ötsch \& F. Rommel (Hrsg.), Spiel-Räume des Denkens. Festschrift zu Ehren von Karl-Heinz Brodbeck (S. 257-286). Marburg: Metropolis.

van Treeck, T. \& Urban, J. (Hrsg.) (2016). Wirtschaft neu denken. Blinde Flecken in der Lehrbuchökonomie. Berlin: iRights Media.

Ulrich, P. (2008). Integrative Wirtschaftsethik. Grundlagen einer lebensdienlichen Ökonomie (4. Aufl.). Bern: Haupt.

Open Access Dieses Kapitel wird unter der Creative Commons Namensnennung 4.0 International Lizenz (http://creativecommons.org/licenses/by/4.0/deed.de) veröffentlicht, welche die Nutzung, Vervielfältigung, Bearbeitung, Verbreitung und Wiedergabe in jeglichem Medium und Format erlaubt, sofern Sie den/die ursprünglichen Autor(en) und die Quelle ordnungsgemäß nennen, einen Link zur Creative Commons Lizenz beifügen und angeben, ob Änderungen vorgenommen wurden.

Die in diesem Kapitel enthaltenen Bilder und sonstiges Drittmaterial unterliegen ebenfalls der genannten Creative Commons Lizenz, sofern sich aus der Abbildungslegende nichts anderes ergibt. Sofern das betreffende Material nicht unter der genannten Creative Commons Lizenz steht und die betreffende Handlung nicht nach gesetzlichen Vorschriften erlaubt ist, ist für die oben aufgeführten Weiterverwendungen des Materials die Einwilligung des jeweiligen Rechteinhabers einzuholen.

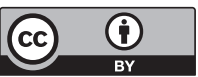

